\title{
Telangiectatic osteosarcoma: a review of literature
}

This article was published in the following Dove Press journal:

OncoTargets and Therapy

27 May 2013

Number of times this article has been viewed

\author{
Jun-jian Liu',* \\ Shen $\mathrm{Liu}^{2, *}$ \\ Jian-guang Wang' \\ Wei Zhu' \\ Ying-qi Hua' \\ Wei Sun' \\ Zheng-dong Cai'
}

'Department of Orthopedic Surgery, Shanghai Tenth People's Hospital,

Tongji University School of Medicine, Shanghai, People's Republic of China;

${ }^{2}$ Department of Orthopedics, the Sixth Affiliated People's Hospital, Shanghai Jiaotong University School of Medicine, Shanghai, People's Republic of China

*These authors contributed equally to this work
Correspondence: Zheng-dong Cai Department of Orthopedic Surgery, Shanghai Tenth People's Hospital, Tongji University School of Medicine, 30 I Yanchang Road, Shanghai, People's Republic of China, 200072 Tel +8602I 66307059

Fax +8602I 66307046

Email czd856@vip.sohu.com

\begin{abstract}
Telangiectatic osteosarcoma is a rare variant of osteosarcoma and hence its occurrence, presentation, and prognosis are poorly understood. With advancements in technology and available treatment options, the scenario of its diagnosis, management, and outcome has changed. Chemotherapy with surgery was challenged previously, but has now been proved to be beneficial. We reviewed the available literature and compared results to define the characteristics of the disease, its presentation, radiographic and pathologic features, optimal treatment, and prognosis.
\end{abstract}

Keywords: telangiectatic osteosarcoma, osteosarcoma, radiology

\section{Introduction}

Telangiectatic osteosarcoma (TO) is one of the rare subtypes of osteosarcoma (OS) and accounts for $2 \%-12 \%$ of all tumors in the appendicular skeleton. ${ }^{1,2} \mathrm{TO}$ is a rare variant of OS with distinctive radiographic, gross, and microscopic features and prognostic implications. The common sites for the tumor location are the fastest growing long tubular bones; the femur is involved most frequently, followed in frequency by the tibia and humerus - a distribution similar to that of conventional OS. ${ }^{2}$ In these bones, the metaphysis is the usual site of origin. ${ }^{3-5}$ Other bones such as the sternum, scapula ribs, innominate bone, cranial vault, and mandible are uncommonly involved. TO of the spine accounts for $2 \%$ of all cases of primary vertebral OS. ${ }^{6}$ This primary highgrade OS of the bone is commonly seen in children, adolescents, and young adults. By contrast, primary OS in atypical locations such as the axial skeleton or soft tissues occurs in middle to late adulthood.

TO is distinguished histologically by spaces, often blood-filled, separated by septa containing highly malignant cells; radiographically, it is distinguished by a predominately lytic and/or expansile component. ${ }^{7,8}$ On radiographs, one can note a very aggressive lesion that is poorly defined and destroys the cortex, often with involvement of the soft tissues. ${ }^{9}$ Similar to other OSs, the telangiectatic variant is diagnosed by radiographic evaluation and biopsy. In early days, TO was diagnosed only when the mesenchymal tumor was found to be characterized by the direct production of osteoid or bone by malignant cells. However, several cases were later reported where bone osteoid was not found. The first detailed report on three cases of telangiectatic extraskeletal OS also noted the absence of osteoid in one case. ${ }^{10}$

Matsuno et $\mathrm{al}^{11}$ proposed the following criteria for the diagnosis of TO: (1) roentgenographically, the lesion shows lytic destructive appearance with no appreciable 
sclerosis; (2) grossly, the lesion is a cavity with little solid tumor mass and no area of sclerosis; and (3) microscopically, the tumor consists of single or multiple cystic cavities that contain blood or necrotic tumor. It is often traversed by septa composed of anaplastic-appearing cells. Malignant cells may be found at the periphery and osteoid production is minimal.

Despite the early recognition ofTO by Paget ${ }^{12}$ in 1853 and its classification as a variant of osteogenic sarcoma in 1922 by Ewing, ${ }^{13}$ there is still disagreement regarding the incidence, clinical behavior, optimal treatment, and prognosis of this entity. The purpose of this review was to systematically study the available literature, compare the results, and define the characteristics of the disease, its presentation, radiographic and pathologic features, optimal treatment, and prognosis.

\section{Is there a role of genetics in the pathogenesis of TO?}

The exact cause of TO is unknown and so are the risk factors. Furthermore, the rare occurrence of the disease makes epidemiological studies difficult and the etiologic factors have not been extensively investigated. An 11-year-old girl and an 8 -year-old boy who were cousins presented with tumors in metaphysis of distal tibia and the lamina of thoracic vertebra, respectively. The tumors were aggressive and destructive but the clinical outcome was favorable in both the cases. The histologic features showed anaplastic tumor cells with osteoid and proliferation of blood cavities. ${ }^{14}$ Mirra et al also described three well-documented cases of pure, extra skeletal TO of the soft tissues, with a "pure" telangiectatic gross and histologic pattern. ${ }^{10}$ The distinctive gross features of the tumors were a predominance of large, blood-filled spaces. On histologic examination, large blood-filled cavities in association with cyst-like walls containing anaplastic spindle cells and definite osteoid production were noted in two of three cases. These cases suggest the presence of genetic factor in the development of TO. ${ }^{10}$

\section{A review of the rare case presentations of TO}

TO most frequently affects the femur, followed in frequency by the tibia and humerus; however, the occurrence of TO at other sites such as the patella, vertebrae, cranial vault, and mandible have also been reported. Chowdhury et $\mathrm{al}^{15}$ reported a case of a 15-year-old girl presenting with complaints of wrist pain and restricted movements of the wrist joint. The patient underwent resection of the distal $8 \mathrm{~cm}$ of the ulna after the confirmatory diagnosis of TO. The patient remained disease free for 69 months after which the TO recurred. There was no evidence of metastatic disease. The forearm was amputated and the patient was aggressively treated with chemotherapy. A follow-up was done for 42 months after her second operation, and no recurrence or metastasis was observed. ${ }^{15}$ The first case of the telangiectatic variant of OS in the jaw bones was reported in a 15-year old Chinese patient by Chan et al. ${ }^{16}$ This patient had a 4-month history of progressive left cheek swelling and the diagnosis was confirmed by biopsy of the resected tumor. Initial excision and subsequent left hemimandibulectomy failed to eradicate the tumor. Radiotherapy and then chemotherapy were given, but the patient showed no improvement. Right proptosis developed and the patient ran a downhill course, dying 16 months after presentation.

Simon and Irwin ${ }^{17}$ described a case of TO in the anterior region of sacrum in a 31-year-old male patient. The tumor was originally thought to be a chordoma and treated surgically, but on pathologic examination was diagnosed as TO. The patient presented with decreased scrotal sensation and difficulty in urination and bowel movement postsurgery, which was subsequently resolved. He did not receive any adjuvant treatment and, after a follow-up of 5 years, was found to have no recurrence or metastasis. ${ }^{17}$ Another unusual case of TO has been published by Shehadeh et al, ${ }^{18}$ wherein a 22-year-old male patient was referred with a history of anterior knee pain for 6 months, which had been originally diagnosed as chondromalacia patellae based on history. Review of anteroposterior and lateral radiographs of the knee demonstrated a diffuse destructive process involving the majority of the patella. A second, noncontiguous lesion (skip lesion) involving the proximal tibia was also observed, with signal characteristics similar to that of the patellar lesion on magnetic resonance imaging (MRI). Based on the histologic findings and imaging studies, the patient was classified as having an Enneking stage 3 (American Joint Committee on Cancer stage 4) metastatic TO with both skip metastases and lung metastases. He received pre- and postoperative chemotherapy and a palliative limb-sparing surgery was done. The patient recovered and resumed activity but long term follow-up was unavailable. ${ }^{18}$

Amritanand et $\mathrm{al}^{19}$ described a rare case of TO of the thoracic spine leading to paraplegia. The patient complained of back pain for 3 months and subsequently developed weakness and numbness of both lower limbs, which over a period of 1 week progressed to complete paraplegia with loss of bowel and bladder function. Histopathological assessment of the specimen showed organizing fibrous tissue, giant cell reaction, and 
osteoid production. Three possible diagnoses were considered - aneurysmal bone cyst (ABC), giant cell tumor (GCT), or a TO. Open biopsy was done and the tissues were sent for a histopathological examination, which revealed a tumor showing multiple, aneurysmally dilated, large blood-filled septate spaces with spindle cells and osteoclast-type multinucleate giant cells. Scattered, markedly pleomorphic tumor cells with hyperchromatic, mitotically-active nuclei were present; in foci, lace-like osteoid admixed with tumor cells and reactive new bone was seen, and a diagnosis of TO was finally made. The patient was advised palliative chemotherapy and radiotherapy. ${ }^{19}$ Another case of spine TO with the involvement of cervical vertebrae has been reported by Turel et al. ${ }^{2}$ The patient presented with a 6-month history of neck pain and torticollis. A cervical spine radiograph showed an expansile bony lesion involving the anterior and posterior elements of the C-5 vertebra with anterolisthesis of C-5 over C-6. The histopathological sample obtained at the first surgery (corpectomy) showed sclerotic cancellous bone with foci of hemorrhage, osteonecrosis, and spindle cell proliferation typical of an $\mathrm{ABC}$. The patient underwent preoperative glue embolization followed by a staged subtotal C-5 spondylectomy and posterior fusion for a C-5 vertebral body lytic expansile lesion. Histopathological examination after the second surgery showed foci of malignant osteoid formation and a diagnosis of TO was made. The surgery was followed by adjuvant radiation and chemotherapy with a favorable outcome at the 1-year follow-up. ${ }^{2}$

A rare case of case of TO involving multiple bones has been reported by Park and Joo. ${ }^{20}$ The patient complained of mild pain and swelling in her foot, occurring over a period of 4 months. A plain X-ray of the foot showed a pure osteolytic lesion with involvement of the navicular, cuboid and 1, 2, and 3 cuneiform bones. On magnetic resonance images, a T1-weighted image showed heterogeneous low signal intensity with involvement of all tarsal bones. A T2-weighted image showed heterogeneous high signal change with multifocal cystic foci and fluid-fluid levels and hence, the diagnosis of multicentric TO was made. The patient underwent a Syme's operation. Histologically, the lesion showed numerous cyst-like spaces divided by multiple septae. The septae were composed of highly atypical sarcomatous tissue, characterized by a high degree of nuclear atypia, cellular pleomorphism, and numerous atypical mitoses. In some microscopic fields, the osteoid-like production was observed in the septae. Postoperatively, the patient received three cycles of chemotherapy and responded well. ${ }^{20}$ Table 1 provides an overview of the case reports of this rare variant of osteosarcoma that has been published in the literature so far.

\section{Extraskeletal TO}

Extraskeletal OS (EOS) is defined as a malignant mesenchymal neoplasm producing neoplastic osteoid or bone, and occurring within the soft tissues free from any underlying bone, as determined radiographically or by surgical inspection. ${ }^{21} \mathrm{TO}$ of the soft tissue is rare subtype of EOS and is characterized by the production of neoplastic osseous tissues, without attachment to bone or the periosteum. It generally affects adults older than 50 years of age. ${ }^{22}$ Dubec et $\mathrm{al}^{23}$ described the clinical and MRI findings in two cases of TO arising in the pretibial soft tissues. Both tumors demonstrated marked inhomogeneity with T2-weighted spin echo and short-tau inversion-recovery sequences. One of the tumors revealed numerous fluid levels within the lesion. Contrary to the above cases where the patients were $>70$ years of age, Sirikulchayanonta and Jaovisidha ${ }^{22}$ reported a case of TO in a teenage male patient who developed a mass in the right thigh. The noncontrasted axial computed tomography (CT) image showed an ill-defined mass that contained a hyperdense area that was likely comprised of blood components. Sagittal T1-weighted MRIs showed mixed hypo- and hypersignal intensity, while axial T2-weighted MRIs showed nonhomogeneous increased signal intensity of the lesion, with multiple fluid-fluid levels indicating multiple locules of blood, and confirmed the diagnosis of TO. The patient responded well to the treatment, showing no signs of relapse. ${ }^{22}$ Another case of rare EOS with telangiectatic features occurring in the mesentery has been described by Lee et al. ${ }^{24}$ The patient was a 67 -yearold male who presented with blood-tinged stool of 1 month's duration. Curative resection of the tumor was performed, and the excised tumor showed large multilocular cysts containing old hematomas and necrotic debris. Histology revealed an OS showing osteoid formation and blood-filled spaces lined with atypical cells. Despite postoperative chemotherapy, he developed a recurrent peritoneal mass and multiple lung metastases 3 months after the surgery. ${ }^{24}$

Van Roggen et $\mathrm{al}^{25}$ described a mesenchymal extraskeletal tumor showing the unequivocal morphology of a TO, arising in the breast in intimate association with a previously excised phyllodes tumor. A 50-year-old patient presented with a painless lump in her right breast. She had undergone local excision of phyllodes tumor twice at this site previously. Sonography revealed a predominantly cystic mass with an irregular luminal outline and numerous fluid levels. Following radiological and cytological investigation, excision was advised. Histology showed focal remnants of the previously excised phyllodes tumor in continuity with areas of widespread differentiation towards a TO. ${ }^{25}$ 


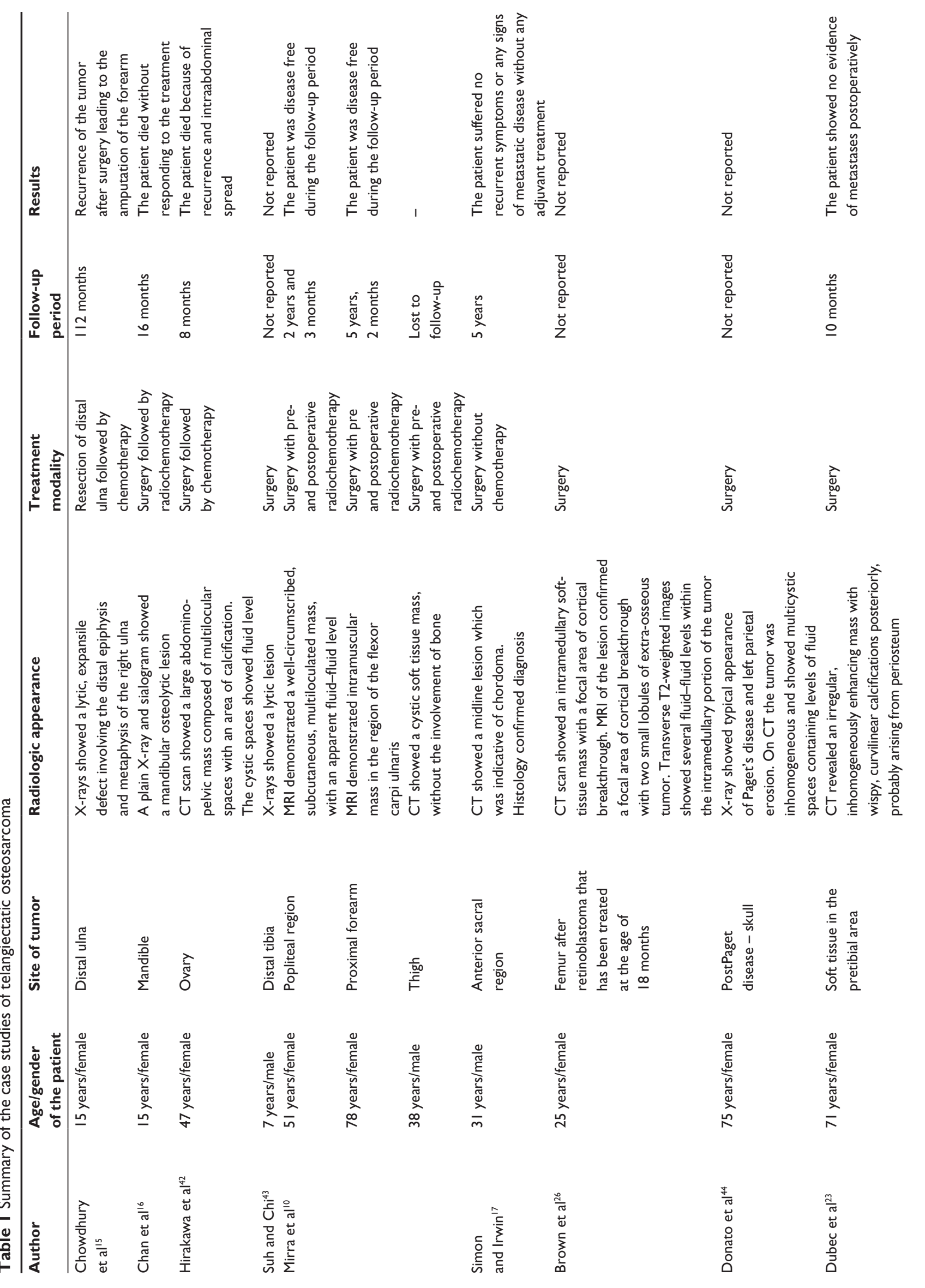



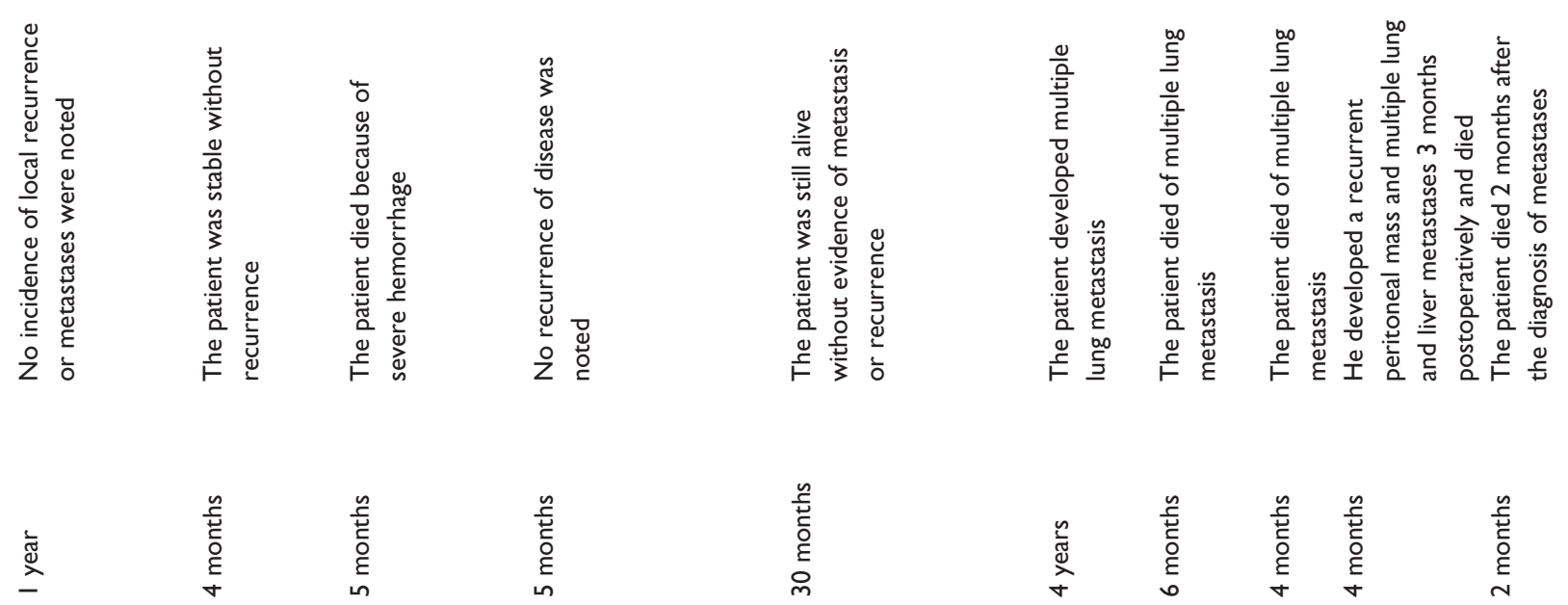

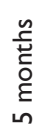

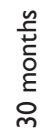

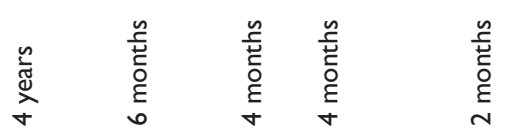

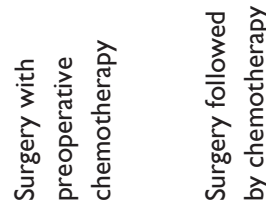
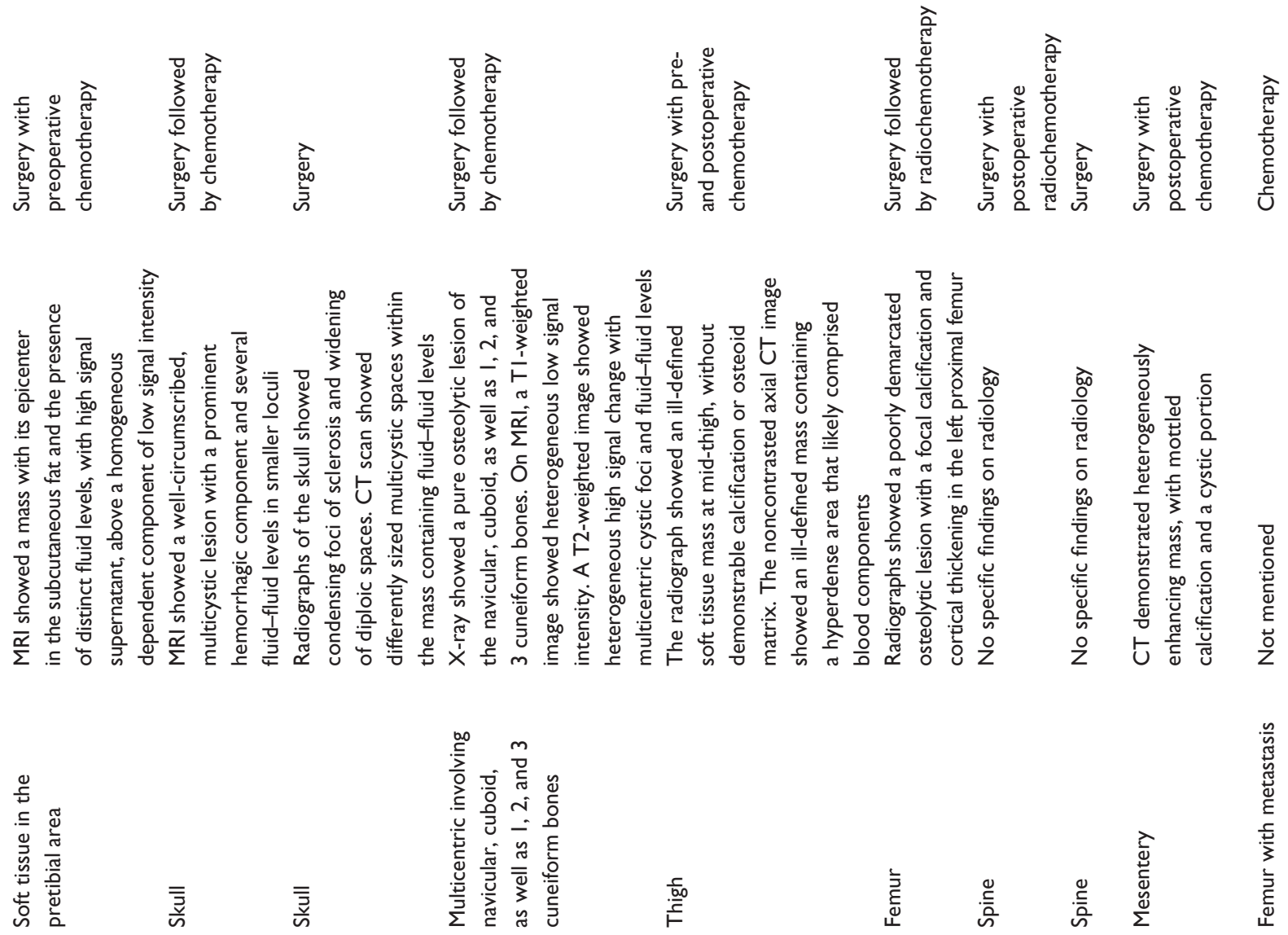

$\frac{\frac{5}{.00}}{\stackrel{5}{F}}$
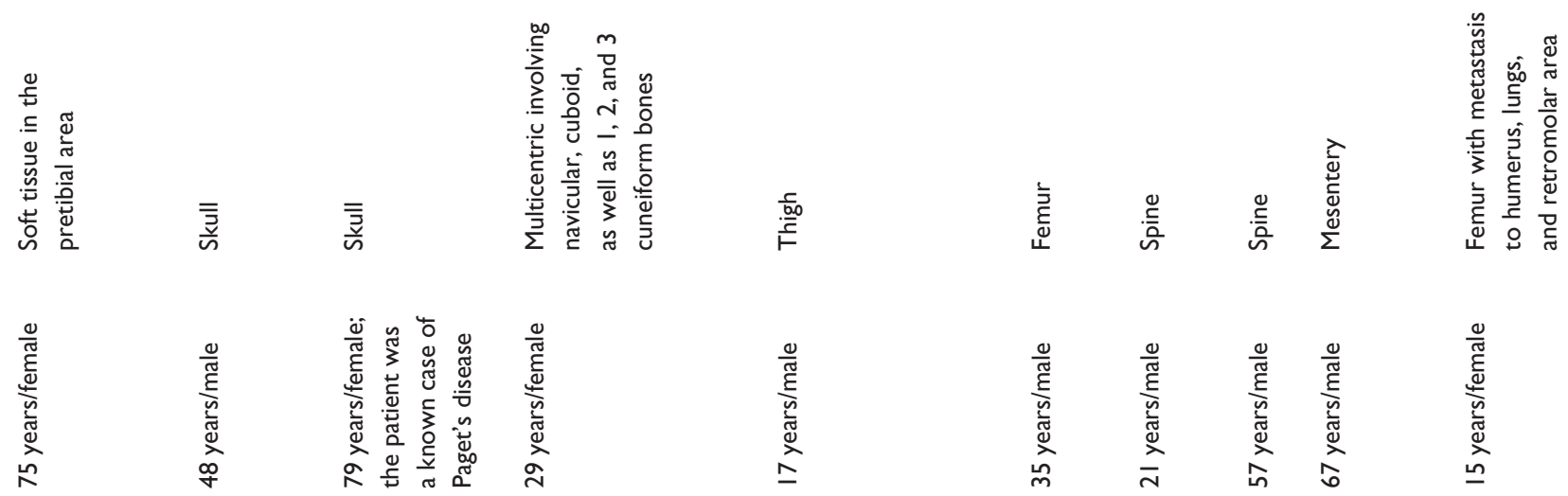

$\frac{0}{\frac{0}{0}}$

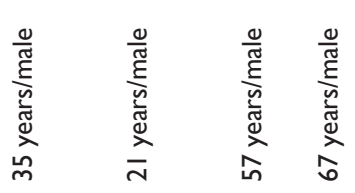

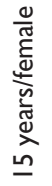
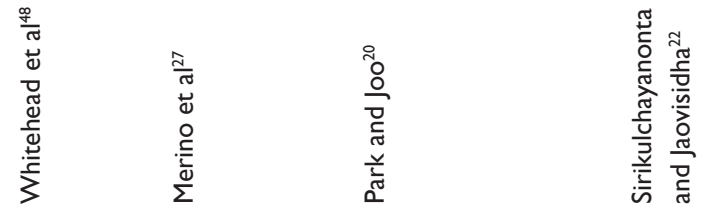

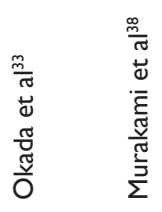

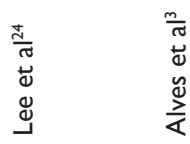




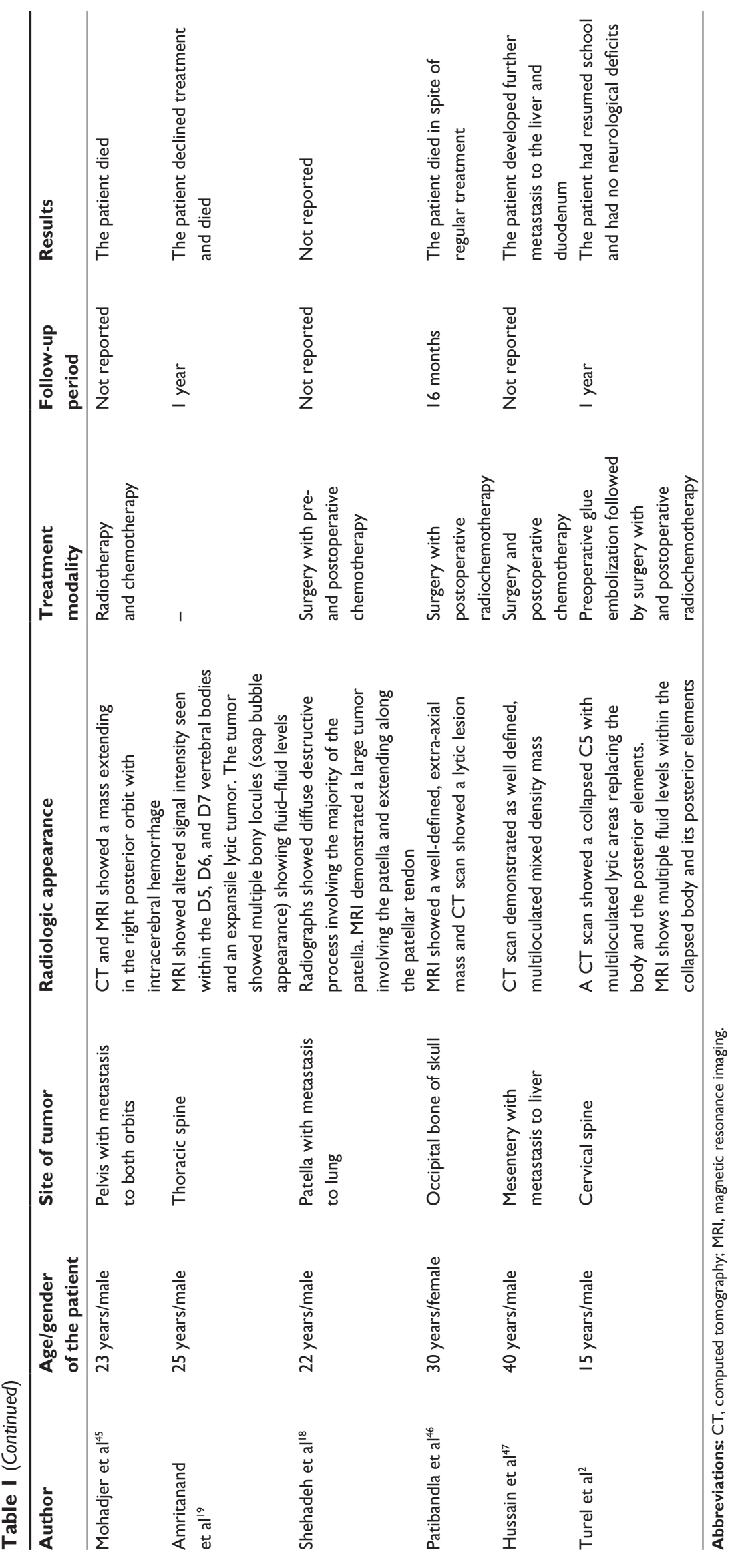




\section{TO in previously diagnosed malignancy cases}

Brown et $\mathrm{al}^{26}$ have reported a unique case in which the patient was diagnosed with TO after successful treatment of his bilateral retinoblastomas 23 years back. The patient had complaints of left knee pain radiating to her groin and inability to walk that lasted for several months in spite of treatment. Past medical history included bilateral enucleation and radiotherapy for bilateral retinoblastomas at the age of 18 months. Plain radiography of the left femur showed a purely lytic, expansile lesion in the mid-diaphysis of the left femur with ill-defined margins, no matrix calcification, and no periosteal reaction. Transverse T2-weighted images on MRI showed several fluid-fluid levels within the intramedullary portion of the tumor. The patient underwent a limb salvage procedure. ${ }^{26}$

Another case study presented the diagnosis and progression of post-Paget TO of the skull, which is also very uncommon. A patient suffering from severe polyostotic Paget's disease over the course of 22 years, presented with a right frontal swelling that had enlarged dramatically in the previous 8 months. The serum alkaline phosphatase concentration was 1537 IU/1 (normal 35-120 IU/1). Radiographs demonstrated Paget's disease of the left scapula, lumbar spine, flat bones of the pelvis, and both femurs. Radiographs of the skull showed condensing foci of sclerosis and widening of diploic spaces. The mass had differently sized multicystic spaces within it, containing fluid-fluid levels as noted on the CT scan. The patient underwent craniectomy and the tumor was partially removed. Chemotherapy was advised as a further line of treatment but the patient declined and she died after 5 months. ${ }^{27}$

\section{Differentiating TO from $A B C$ : a clinical challenge}

TO can very often be radiologically confused with $\mathrm{ABC}$ or $\mathrm{GCT}^{28,29} \mathrm{CT}$ and MRI findings of TO often include thick nodular tissue (and matrix mineralization at $\mathrm{CT}$ ) in a largely hemorrhagic and/or necrotic osseous lesion with an associated soft-tissue mass, which allows distinction from $\mathrm{ABC} .{ }^{30}$ However, the diagnosis is still confusing and several cases are available in literature where the diagnosis of $\mathrm{ABC}$ was ruled out to confirm the presence of TO.

The classic example is a case of a 20 -year-old man with $\mathrm{TO}$ of the femur which was initially diagnosed and managed as ABC. The lesion recurred twice. At first, recurrence MRI showed a multilocular low-intensity lesion on the T1-weighted images and high intensity lesion on the
T2-weighted images. The clinical diagnosis for this lesion was recurrence of $\mathrm{ABC}$, and curettage and bone graft with internal fixation was performed. At the second recurrence, CT scan revealed cortical destruction with a large soft tissue component. The patient underwent wide resection of the tumor with prosthetic replacement of the right proximal femur. The histologic section for this lesion showed a bloodfilled cystic lesion with sarcomatous cells in its wall. The cells showed features of atypical mitoses and tumor osteoid; a diagnosis of TO was made. ${ }^{31}$

\section{Dedifferentiation of other tumors into TO}

Dedifferentiated chondrosarcoma with features of TO is very rare and the literature mentions only two such cases so far. A case of dedifferentiated chondrosarcoma with TO-like features in a 44-year-old female has been presented by Radhi and Loewy. ${ }^{32}$ The patient had a history of solitary osteochondroma of the scapula for 5 years which later developed into an aggressive lesion. X-ray of the chest and scapula revealed a cauliflower-like growth with lytic areas and similar findings were reported on CT scan. Excision was performed and the pathological examination confirmed the diagnosis of TO. Exposure to X-rays was thought to be an aggravating factor. ${ }^{32}$

In another such case, a 35-year-old male Chinese patient reported with a 2-month history of pain around the left thigh. Radiographs showed a poorly demarcated osteolytic lesion with focal mineralization and endosteal scalloping in the left proximal femur. Biopsy showed a proliferation of highly anaplastic cells without any cartilaginous component. A wide excision of the left proximal femur with a replacement by endoprosthesis was carried out after treatment with methotrexate and 20 Gy radiation therapy. Pathological examination of the surgical specimen showed a focus of low-grade chondrosarcoma and the coexistence of TO-like features. The patient was diagnosed with dedifferentiated chondrosarcoma with TO-like features. ${ }^{33}$ In both these cases, the patient's age was less than usual (chondrosarcoma usually occurs in patients aged $>50$ years), suggesting that young people may be uniquely prone to this unusual variant of dedifferentiated chondrosarcoma.

As with chondrosarcoma, dedifferentiation has also been observed in cases of parosteal OS (POS). Azura et al $1^{34}$ performed a study to assess the imaging findings in cases of POS dedifferentiated into TO. A systemic review of 199 patients diagnosed as surface OS was performed. Eightysix patients with POS were identified and among them, 
23 were found to have a dedifferentiated lesion. Histology confirmed three cases to have TO. ${ }^{34} \mathrm{~A}$ case of POS of the proximal femur, with areas of telangiectatic dedifferentiation, in a 28-year-old woman has been reported by Wines et al. ${ }^{35}$ The patient presented with a 7-week history of discomfort and swelling in the anterior aspect of her right thigh. Radiographs showed a large ossified mass attached to the proximal femur, with destruction of the adjacent bony cortex. On the basis of the histological and radiological findings, a diagnosis of POS was made. The tumor was resected and the patient also underwent chemotherapy. When the excised tumor was studied, the wall of one cyst contained pleomorphic stromal cells with atypical nuclei, which are associated with osteoid production. A diagnosis of localized TO dedifferentiation was made. The patient died because of metastasis to lung and gluteal region. ${ }^{35}$

\section{Management of TO}

The prognosis of TO was initially thought to be poor; however, the scenario has changed and it has been found that TO responds well to the treatment. ${ }^{36,37}$ In addition to surgical excision, adjuvant chemotherapy plays an important role in the treatment of TO. Murakami et a ${ }^{138}$ reported en bloc tumor excision, including the spinal cord in two cases for TO. In one case, radiochemotherapy was used as an adjuvant treatment, while in the other case the patient declined chemotherapy. Both the patients had complete paraplegia when they presented to the cancer center; hence, complete segmental spinal resection (total en bloc spondylectomy) was performed. However, the patients succumbed to the disease and died of lung metastasis. ${ }^{40}$

In another study, Bacci et al $^{37}$ treated 24 patients with neoadjuvant chemotherapy using two protocols. The patients were known cases of TO of the extremities. Twenty-one patients underwent limb salvage; amputation and rotation plasty was done in the other three. The patients were followed up for a mean duration of 74 (60-96) months, and it was noted that 20 patients remained continuously free of disease and four relapsed with lung metastases. There were no local recurrences. The study compared these results with the ones achieved in 269 contemporary patients with conventional OS of the extremities using the same protocols for chemotherapy. They observed that there was a significantly better histologic response to chemotherapy (96\% versus $68 \%$ of good histologic response; $P=0.004$ ) and disease-free survival $(83 \%$ versus $55 \% ; P=0.01)$ in the TO group. The study concluded that TO was more sensitive to chemotherapy than conventional OS and that most of these patients may be cured without amputation. ${ }^{37}$ A similar study was done by one other group of researchers and similar results were demonstrated. Twenty-eight patients with telangiectatic osteogenic sarcoma of the extremities were treated with neoadjuvant chemotherapy between March 1983 and March 1990. A good histological response (tumor necrosis $>90 \%$ ) was observed in 25 patients ( $89 \%$ ) and at a mean follow-up of 5 years ( $2-9$ years) 23 patients ( $82 \%$ ) remained continuously disease-free and five developed lung metastases. ${ }^{39}$

Weiss et al ${ }^{36}$ reviewed the patients with $\mathrm{TO}$ and observed the same response. Estimates of 5-year event-free survival $(58.3 \% \pm 11.9 \%)$ and overall survival $(66.8 \% \pm 11.6 \%)$ were similar to those for patients with other OS subtypes $(P>0.85)$. The absence of local disease progression and chemotherapy with more than three agents that were active against OS were correlated with improved outcome $(P \leq 0.005){ }^{36}$ Mervak et $\mathrm{l}^{40}$ also reported the efficacy of adjuvant chemotherapy in improving the prognosis. They remarked that chemotherapy is particularly important in patients with metastasis; in patients without metastasis, survival was not influenced by whether they received chemotherapy or not.

\section{Discussion}

TO is a rare, aggressive subtype of OS characterized pathologically by large cavities filled with fresh and clotted blood. The tumor has distinctive roentgenographic, gross, and microscopic features. Although conventional OSs may contain telangiectatic elements, only those composed almost entirely of telangiectatic tissue are generally considered true examples of this entity. In our review, we found two cases of dedifferentiated chondrosarcoma with TO-like features and a case of dedifferentiated parosteal OS with TO-like features. Dedifferentiation of chondrosarcoma and parosteal OS into a more aggressive lesion is frequent and usually visible on imaging; hence, a diagnosis of TO should be ruled out when managing such patients.

TOs have a skeletal and age distribution similar to conventional central OSs and are most commonly found in the metaphysis of long bones, although they may arise within the diaphysis. We have reviewed the cases of TO in several unusual locations like the mandible, skull, sacrum, patella, and one case of multicentric involvement affecting navicular, cuboid, as well as 1,2, and 3 cuneiform bones. Apart from these, our review also highlights the presence of the tumor in soft tissues like mesentery, breast, and ovaries; therefore, surgeons and radiologists should not rule out a possibility of $\mathrm{TO}$ in these areas without proper radiologic and histologic examination. 
Our review confirms the criteria for diagnosis of TO which includes: (1) radiographically, a destructive, osteolytic tumor with little or no matrix mineralization or periosteal new bone formation; (2) a cystic, cavitary gross specimen with little solid tumor tissue and no areas of dense bone; and (3) histologically, a tumor with single or multiple cystic cavities, containing blood or necrotic tissue, with septa composed of anaplastic tumor cells. The radiographic findings of geographic margins, cortical bone destruction, and absence of matrix calcification may resemble benign lesions such as GCT or ABC. TO also shares certain clinical, radiologic, and pathologic features with $\mathrm{ABC}$ and it is therefore often difficult to distinguish between these two lesions, especially in their early stage. Although malignant transformation of $\mathrm{ABC}$ has been reported, virtually all such cases represent either radiation induced sarcomas or TOs that were initially misdiagnosed as an ABC. Therefore, proper differentiation and diagnosis should be made using a CT or MRI.

On MRI, TO frequently exhibit high-signal intensity regions on T1-weighted images due to the presence of methemoglobin within the hemorrhagic spaces. Fluid-fluid levels on MRI are a nonspecific finding that may be seen in aneurysmal cysts, GCTs, fibrous dysplasia, and unicameral bone cysts. However, the presence of fluid-fluid levels in the calvarial mass and the associated finding of a Pagetic skull were important clues to the diagnosis of TO in Paget's disease in one of the case that we reviewed. Another important aspect of this case was an increase in the alkaline phosphatase levels, which has a diagnostic importance in TO. Another case study described by Yoshida et $\mathrm{al}^{41} \mathrm{dem}$ onstrated that alkaline phosphatase activity in biopsy and surgical specimens is useful for distinguishing TOs from other osteolytic bone tumors.

The available literature in early studies described a poor prognosis for patients with TO. However, more recent work employing neoadjuvant chemotherapy with surgery has demonstrated survival rates for TO to be better than conventional OS. ${ }^{42}$ The 5-year survival rate for TO of the bone has shown a dramatic response to chemotherapy, appearing to be more sensitive to chemotherapy than conventional OS. ${ }^{43}$ However, if treated only with surgery, the prognosis is even worse than that of conventional OS. Studies by Bacci et $\mathrm{al}^{37}$ and Weiss et $a l^{36}$ support the use of chemotherapy with surgery in the patients of TO. In a few of the cases reviewed, surgery was accompanied by pre and post chemotherapy, while a few cases also received radiation therapy. Of the 22 case studies that we studied, nine patients died; the nine patients had either declined treatment or were in the late stage of disease with metastases to several organs. Early diagnosis and prompt treatment was important for disease-free survival.

\section{Disclosure}

The authors report no conflicts of interest in this work.

\section{References}

1. Yarmish G, Klein MJ, Landa J, Lefkowitz RA, Hwang S. Imaging characteristics of primary osteosarcoma: nonconventional subtypes. Radiographics. 2010;30(6):1653-1672.

2. Turel MK, Joseph V, Singh V, Moses V, Rajshekhar V. Primary telangiectatic osteosarcoma of the cervical spine. J Neurosurg Spine. 2012;16(4):373-378.

3. Alves FA, Lopes MA, Ikeda MK, Kowalski LP, Almeida OP. Oral metastasis of telangiectatic osteosarcoma. Oral Dis. 2003;9(2):104-106.

4. Bloem JL, Kroon HM. Osseous lesions. RadiolClin North Am. 1993 31(2):261-278.

5. Seeger LL, Gold RH, Chandnani VP. Diagnostic imaging of osteosarcoma. ClinOrthopRelat Res. 1991;270:254-263.

6. Ilaslan H, Sundaram M, Unni KK, Shives TC. Primary vertebral osteosarcoma: imaging findings. Radiology. 2004;230(3):697-702.

7. Dorfman H, Bogdan C. Bone Tumors. St Louis, MO: Mosby; 1998.

8. Sternberg S. Diagnostic Surgical Pathology. Philadelphia, PA: Lippincott, Williams, \& Wilkins; 1999.

9. Vanel D, Tcheng S, Contesso G, et al. The radiological appearances of telangiectatic osteosarcoma. A study of 14 cases. Skeletal Radiol. 1987;16(3)196-200.

10. Mirra JM, Fain JS, Ward WG, Eckardt JJ, Eilber F, Rosen G. Extraskeletal telangiectatic osteosarcoma. Cancer. 1993;71(10):3014-3019.

11. Matsuno T, Unni KK, McLeod RA, Dahlin DC. Telangiectaticosteogenic sarcoma. Cancer. 1976;38(6):2538-2547.

12. Paget J. Lectures on Surgical Pathology. London: Longmans, $1853 ; 2: 281$

13. Ewing J. A review and classification of bone sarcomas. Arch Surg. 1922;4:485-533.

14. Nishida J, Abe M, Shiraishi H, et al. Familial occurrence of telangiectatic osteosarcoma: cousin cases. J PediatrOrthop. 1994;14(1):119-122.

15. Chowdhury K, Bachynski B, Alport EC. Telangiectatic osteosarcoma: unusual behaviour. Can J Surg. 1986;29(1):29-31.

16. Chan CW, Kung TM, Ma L. Telangiectatic osteosarcoma of the mandible. Cancer. 1986;58(9):2110-2115.

17. Simon RG, Irwin RB. An unusual presentation of telangiectatic osteosarcoma. Am J Orthop (Belle Mead NJ). 1996;25(5):375-379.

18. Shehadeh AM, Haiba MA, Henshaw RM, Lack E. Telangiectatic osteosarcoma of the patella. Orthopedics. 2008;31(8):808.

19. Amritanand R, Venkatesh K, Cherian R, Shah A, Sundararaj GD. Telangiectatic osteosarcoma of the spine: a case report. Eur Spine J. 2008;17(Suppl 2):S342-S346.

20. Park YK, Joo M. Multicentrictelangiectatic osteosarcoma. Pathol Int. 2001;51(3):200-203.

21. Enzinger FM, Weiss SW, editors. Soft Tissue Tumors. 3rd ed. New York: CV Mosby; 1995.

22. Sirikulchayanonta V, Jaovisidha S. Soft tissue telangiectatic osteosarcoma in a young patient: imaging and immunostains. Skeletal Radiol. 2005;34(5):295-298.

23. Dubec JJ, Munk PL, O'Connell JX, et al. Soft tissue osteosarcoma with telangiectatic features: MR imaging findings in two cases. Skeletal Radiol. 1997;26(12):732-736.

24. Lee KH, Joo JK, Kim DY, Lee JS, Choi C, Lee JH. Mesenteric extraskeletal osteosarcoma with telangiectatic features: a case report. BMC Cancer. 2007; 7:82.

25. Graadt van Roggen JF, Zonderland HM, Welvaart K, Peterse JL, Hogendoorn PC. Local recurrence of a phyllodestumour of the breast presenting with widespread differentiation to a telangiectatic osteosarcoma. J ClinPathol. 1998;51(9):706-708. 
26. Brown MJ, Logan PM, O’Connell JX, Janzen DL, Connell DG. Diaphysealtelangiectatic osteosarcoma as a second tumor after bilateral retinoblastomas. Skeletal Radiol. 1996;25(7):685-688.

27. Merino S, Arrazola J, Saiz A, Blanco JA, Ortega L. Post-Paget telangiectatic osteosarcoma of the skull. Skeletal Radiol. 1999;28(8):470-472.

28. Roger DJ, Uhl RL, Carl A. Malignant fibrous histiocytoma of the patella. Orthopedics. 1994;17(2):189-193.

29. Kransdorf MJ, Moser RP Jr, Vinh TN, Aoki J, Callaghan JJ. Primary tumors of the patella: A Review of 42 cases. Skeletal Radiol. 1989;18(5): 365-371.

30. Murphey MD, wan Jaovisidha S, Temple HT, Gannon FH, Jelinek JS, Malawer MM. Telangiectatic osteosarcoma: radiologic-pathologic comparison. Radiology. 2003;229(2):545-553.

31. Saito T, Oda Y, Kawaguchi K, et al. Five-year evolution of a telangiectatic osteosarcoma initially managed as an aneurysmal bone cyst. Skeletal Radiol. 2005;34(5):290-294.

32. Radhi JM, Loewy J. Dedifferentiated chondrosarcoma with features of telangiectatic osteosarcoma. Pathology. 1999;31(4):428-430.

33. Okada K, Hasegawa T, Tateishi U, Endo M, Itoi E. Dedifferentiated chondrosarcoma with telangiectatic osteosarcoma-like features. J Clin Pathol. 2006;59(11):1200-1202.

34. Azura M, Vanel D, Alberghini M, Picci P, Staals E, Mercuri M. Parosteal osteosarcoma dedifferentiating into telangiectatic osteosarcoma: importance of lytic changes and fluid cavities at imaging. Skeletal Radiol. 2009;38(7):685-690.

35. Wines A, Bonar F, Lam P, McCarthy S, Stalley P. Telangiectatic dedifferentiation of a parosteal osteosarcoma. Skeletal Radiol. 2000;29(10):597-600.

36. Weiss A, Khoury JD, Hoffer FA, et al. Telangiectatic osteosarcoma: the St Jude Children's Research Hospital's experience. Cancer. 2007;109(8):1627-1637.

37. Bacci G, Ferrari S, Ruggieri P, et al. Telangiectatic osteosarcoma of the extremity: neoadjuvant chemotherapy in 24 cases. ActaOrthop Scand. 2001;72(2):167-172.
38. Murakami H, Tomita K, Kawahara N, Oda M, Yahata T, Yamaguchi T. Complete segmental resection of the spine, including the spinal cord, fortelangiectatic osteosarcoma: a report of 2 cases. Spine (Phila Pa 1976). 2006;31(4):E117-E122.

39. Bacci G, Picci P, Ferrari S, Sangiorgi L, Zanone A, Brach del Prever A. Primary chemotherapy and delayed surgery for non-metastatic telangiectatic osteosarcoma of the extremities. Results in 28 patients. Eur $J$ Cancer. 1994;30A(5):620-626.

40. Mervak TR, Unni KK, Pritchard DJ, McLeod RA. Telangiectatic osteosarcoma. Clin Orthop Relat Res. 1991;270:135-139.

41. Yoshida H, Adachi H, Naniwa S, Yumoto T, Morimoto K, Furuse K. High alkaline phosphatase activity of telangiectatic osteosarcoma (TOS) and its diagnostic significance. ActaPatholJpn. Feb 1987;37(2): 305-313.

42. Hirakawa T, Tsuneyoshi M, Enjoji M, Shigyo R. Ovarian sarcoma with histologic features of telangiectatic osteosarcoma of the bone. Am J Surg Pathol. 1988;12(7):567-572.

43. Suh YL, Chi JG. Telangiectatic osteosarcoma - a case report. J Korean Med Sci. 1989;4(2):97-101.

44. Donato G, Lavano A, Volpentesta G, et al. Telangiectatic osteosarcoma of the skull. A post-Paget case. ClinNeuropathol. 1997;16(4): 201-203.

45. Mohadjer Y, Wilson MW, Fuller CE, Haik BG. Primary pelvic telangiectatic osteosarcoma metastatic to both orbits. Ophthal PlastReconstr Surg. 2004;20(1):77-79.

46. Patibandla MR, Uppin SG, Thotakura AK, Panigrahi MK, Challa S. Primary telangiectatic osteosarcoma of occipital bone: a case report and review of literature. Neurol India. 2011;59(1):117-119.

47. Hussain MI, Al-Akeely MH, Alam MK, Jasser NA. Extraskeletal osteosarcoma, telangiectatic variant arising from the small bowel mesentery. Saudi Med J. 2011;32(9):958-961.

48. Whitehead RE, Melhem ER, Kasznica J, Eustace S. Telangiectatic osteosarcoma of the skull base. AJNR Am J Neuroradiol. Apr 1998; 19(4):754-757.
OncoTargets and Therapy

\section{Publish your work in this journal}

OncoTargets and Therapy is an international, peer-reviewed, open access journal focusing on the pathological basis of all cancers, potential targets for therapy and treatment protocols employed to improve the management of cancer patients. The journal also focuses on the impact of management programs and new therapeutic agents and protocols on

\section{Dovepress}

patient perspectives such as quality of life, adherence and satisfaction The manuscript management system is completely online and includes a very quick and fair peer-review system, which is all easy to use. Visit http://www.dovepress.com/testimonials.php to read real quotes from published authors. 